\title{
Liderazgo pastoral y mayordomía de ingresos económicos de la Mi- sión Centro Oeste del Perú, 2012-2013
}

\author{
Pastoral leadership and stewardship of economic income of the Mis- \\ sion Center West of Peru, 2012-2013
}

\author{
Jorge Alberto Montero Guerrero *1 \\ ${ }^{1}$ Escuela de Posgrado de Administración, Universidad Peruana Unión
}

INFORMACIÓN DEL ARTÍCULO

Historia del artículo:

Recibido el 6 de marzo de 2015

Aceptado el 6 de mayo de 2015

\section{Keywords:}

Liderazgo pastoral

Mayordomía

Ingresos económicos

Desarrollo

Crecimiento

\section{Resumen}

Los cambios que vienen ocurriendo a lo largo de los últimos años en las organizaciones trajeron a discusión la importancia del liderazgo ejercido por las personas y la influencia que pueda tener en su desarrollo y crecimiento. La Iglesia Adventista no es ajena a esta realidad; por ello, la presente investigación estableció como objetivo determinar la influencia del liderazgo del pastor en la mayordomía de ingresos económicos de la Misión Centro Este del Perú (MiCOP), 2012-2013. La metodología fue de tipo cuantitativo, su diseño es transversal de tipo descriptivo correlacional. Se aplicó el instrumento "Cuestionario Multifactorial de Liderazgo" elaborado por Bernard Bass y Bruce Avolio de la Universidad de Binghamton en Nueva York. Este instrumento se aplicó en diversos países y en distintos idiomas. El proceso de recolección de datos se realizó con la autorización de la Junta Administrativa de la MiCOP. Adicionalmente se realizó una reunión con los administradores del campo (focus group), recabándose información respecto del estilo de liderazgo que, en opinión de los administradores, tienen sus pastores distritales. Se pudo determinar que existe una relación significativa entre el estilo de liderazgo de los pastores distritales y los mayordomía de ingresos económicos de la MiCOP, 2012-2013

\begin{abstract}
Abstrac
The changes that have occurred over the past years in the organizations brought to discussion the importance of leadership exercised by the people and the influence that may have on its development and growth. The Seven Day Adventist Church is no stranger to this reality, this motivates this research. Is for this reason that the present study established the following objective: to determinate the influence of pastor's leadership in the stewardship of economic income of the MiCOP, 2012-2013. The methodology was qualitative, its design is transversal descriptive correlational. Applied instrument "Multifactor leadership questionnaire" developed by Bernard Bass and Bruce Avolio at Binghamton University in New York. This instrument was applied in different countries and in different languages. The process of data collection was carried out with the authorization of the Administrative Board of the Mission Center West of Perú MiCOP. Additionally a meeting was held with the managers of the field (focus group), collecting information regarding leadership style
\end{abstract}

\footnotetext{
* Autor para correspondencia: Av. Nicolás Ayllón 200, Chaclacayo, Lima-Perú. Unión Peruana, Perú Correo electrónico: jorge.montero@adventistas.org.pe
} 
which in the opinion of the administrators have their district pastors. It was determined that there is a significant relationship between the style of leadership of the district pastors and the stewardship of economic income of the MiCOP, 2012-2013

\section{Introducción}

El contexto histórico y social representan, actualmente, grandes desafíos para las organizaciones. A fines del 2013, el Foro Económico Mundial presentó una lista de desafíos a enfrentar durante el presente siglo, algunos de ellos son el conflicto en Medio Oriente, la desigualdad económica, el desempleo, el cambio climático, las amenazas cibernéticas.

El desafío que merece acentuada atención se refiere a los líderes y sus valores. Es una preocupación, a todo nivel de la sociedad, encontrar personas con valores, que ejerzam un liderazgo que repercuta positivamente en los resultados. Debemos reconocer que el logro de los resultados depende de una gran cantidad de factores; sin embargo, los estudios actuales señalan que el liderazgo es determinante para alcanzarlos. Bass y Avolio (1993) realizaron un estudio empírico en que se identifican los estilos de liderazgo frecuente de los jefes y comandantes. Se encuentran las dos categorías: liderazgo transformacional y transaccional. Este modelo se considera la gama completa de liderazgo.

Un líder transformacional se centra en "transformar" a otros, a ayudarse mutuamente, a mirar por los demás, a mirar la organización como un todo. En este mandato, el líder aumenta la motivación, la moral y el rendimiento de su grupo de seguidores.

En el Perú la Iglesia Adventista del Séptimo Día, no está ajena a estos desafíos y también como organización debe encontrar la mejor respuesta para enfrentar estos retos, por lo cual es importante identificar el estilo de liderazgo que sus pastores distritales tienen, así como el efecto que este liderazgo tiene sobre los ingresos de la iglesia. Uno de los campos administrativos que más creció en la Unión Peruana del Norte (UPN) es la Misión Centro Oeste del Perú (MiCOP), por lo cual nuestra investigación se centrará en analizar la influencia del liderazgo pastoral en la mayordomía de ingresos económicos de la MiCOP.

Actualmente, las organizaciones sin excepción se encuentran en el centro de un conjunto de expectativas relacionadas con los cambios acelerados, profundos e inevitables, en respuesta al avance y el desarrollo de las tecnologías de información; en respuesta a la celeridad del fenómeno llamado: globalización, también a la celeridad de cambio y la rapidez de las telecomunicaciones. Las organizaciones viven ancladas en este contexto; por consiguiente, tienen el obligado compromiso de responder a estos cambios mediante el liderazgo, la innovación y la creatividad constante; dicha situación obliga, de modo imperativo, a los responsables el ejercicio de una buena dirección, excelente liderazgo y conducción adecuada de las organizaciones. White (1961) meniona que la organización correcta es de Dios; se basa en principios divinos: "El sistema y el orden se manifiestan en todas las obras de Dios a través del universo".

La Iglesia Adventista del Séptimo Día (IASD) es una organización internacional, cuya obra principal está avocada en cumplir la misión encargada por Dios en la Bi- 
blia. La organización de la iglesia sigue un estilo administrativo representativo, pues reconoce que la autoridad de la iglesia descansa en sus miembros y debe ser expresada a través de representantes debidamente escogidos en cada nivel de la organización, cada uno con responsabilidad ejecutiva y administrativa; los niveles de IASD son: iglesia local, Asociación o Misión/campo local, Unión de iglesias, Unión-Asociación o Unión-Misión, Asociación General y sus divisiones.

La Asociación General de la IASD (2000) afirma que sin organización, ninguna institución o movimiento puede prosperar. Una nación sin gobierno organizado no tardaría en hundirse en el caos. Una empresa comercial sin organización fracasaría. Así ocurría con la iglesia; sin la organización se desintegraría y perecería.

\section{Liderazgo}

Muchos estudios se han referido al liderazgo ya sea de forma directa 0 indirecta; así mismo, han sido numerosos los intentos de conceptualizarlo, por lo que existen disímiles definiciones acerca del tema.

El liderazgo, según Fleishman (1991), inplica la influencia que ejerce una persona sobre un grupo de su referencia. Dicha influencia del líder logra que otros lleven a cabo las tareas que se pretenden desarrollar al servicio de la eficacia colectiva. Boudicht (1989) define el liderazgo como el "esfuerzo que se efectúa para influir en el comportamiento de los otros o para ordenar que se alcancen los objetivos organizacionales, individuales o personales".

Covey (1989) refiere que el "liderazgo es primordialmente una actividad que absorbe la energía del cerebro derecho, tiene mucho de arte y se basa en una filosofía". El mismo Covey (2004) sustenta que el "liderazgo consiste en transmitir a las personas su valía y potencial de un modo tan claro que lleguen a verlas en sí mismas. Las personas deben albergar un sentimiento intrínseco de valía, totalmente independiente de la comparación con los demás, y sentir que son merecedores de amor incondicional, independiente de su comportamiento y de su rendimiento".

Chiavenato (1993) menciona que el "liderazgo es la influencia interpersonal ejercida en una situación, dirigida a través del proceso de comunicación humana a la consecución de uno o diversos objetivos específicos".

Para Warren (1994) el liderazgo sostiene que la mayor parte de las organizaciones se encuentran sobreadministrados y subliderados. Una persona quizás sea un gerente eficaz (buen planificador y administrador), justo y organizado, pero carente de las requeridas habilidades de líder comprometido con la motivación. Otras personas tal vez sean líderes eficaces, con habilidad para desatar el entusiasmo y la devolución, pero carece de las habilidades administrativas para canalizar la energía que desatan en otros. Ante los desafíos del compromiso dinámico del mundo actual de las organizaciones, muchas de ellas están apreciando más a los gerentes que también tienen habilidades de líderes.

Senge (2003) argumenta que la nueva visión del liderazgo en las organizaciones inteligentes se enfoca en tareas más sutiles e importantes; los líderes son diseñadores, mayordomos y maestros, son responsables de construir organizaciones cuyo personal expande continuamente su aptitud para comprender la complejidad, clarificar la visión y mejorar los modelos mentales compartidos, es decir, son responsables de aprender. 


\section{Teoría de los rasgos}

Según Adams (1985), el líder posee algunos rasgos específicos de personalidad, gracias a los cuales el líder se distingue de las demás personas, es decir, las características marcadas de personalidad marcan y determinan la influencia en el comportamiento de sus semejantes.

Según los investigadores, las teorías de rasgo parten del supuesto de que ciertos individuos poseen una combinación especial de rasgos de personalidad definidos y utilizados para identificar líderes potenciales. Diversos investigadores plantearon los principales rasgos: rasgos físicos (energía, apariencia, estatura y peso), los rasgos intelectuales (adaptabilidad, entusiasmo y autoestima), rasgos sociales: cooperación, habilidades interpersonales y habilidades administrativas; rasgos relacionados con el trabajo: interés en la realización, persistencia e iniciativa. Dentro de esta teoría, se considera cinco dimensiones: emocional, empatía, ajuste, escrupulosidad, apertura.

Estas teorías de rasgos presentan algunas limitaciones, no ponderan la importancia de cada una de las diversas características y rasgos de personalidad que destacan los aspectos del liderazgo, además olvidan la influencia y reacción de los subordinados en los resultados del liderazgo; no distinguen los rasgos pertinentes para lograr cierto tipo de objetivos, ignoran por completo la situación en la cual se ejerce el liderazgo; esta teoría manifiesta que un individuo dotado de rasgos de liderazgo será líder siempre y en cualquier situación. Por estas razones, estos planteamientos sobre el liderazgo perdieron crédito y dejaron de ser importantes.

\section{El modelo de contingencia}

Fiedler, citado por Robbins (1999), demuestra que el desempeño eficaz del grupo depende de un ajuste adecuado, según la forma como interactúa el líder con sus subordinados y en la medida que la situación le da control e influencia al líder. El estilo de liderazgo de las personas es innato, y no se puede cambiar el estilo para ajustarlos de acuerdo con una situación. Asimismo, considera tres dimensiones de contingencia que determinan la eficacia del liderazgo: la relación líder miembro, la estructura de la tarea y la posición de poder concedido al líder por el puesto que este ocupa. La primera define el grado de credibilidad, confianza y respeto de los subordinados en el líder; la segunda refiere el grado de la tarea del puesto que implica procedimientos estructurados y no estructurados; la tercera mide el grado de influencia del líder sobre las variables de poder: las contrataciones, los despidos, la disciplina, los ascensos y los incrementos salariales. Luego de evaluar la situación en función de las tres variables de contingencias, considera que la relación líder miembro puede ser buena o mala, la estructura de la tarea puede ser alta o baja, y la posición de poder puede ser fuerte o débil. Además argumenta que el líder tendrá más influencia y poder, si mejores son las relaciones con sus subordinados, si las tareas están altamente estructuradas y si la posición de poder es fuerte.

\section{La teoría situacional}

Paul Hersey y Kenneth Blanchard, citado por Robbins (1999), proponen la teoría de contingencia enfocada en la disponibilidad de los seguidores, se refiere a la medida de la capacidad y la voluntad para llevar a cabo las tareas; la teoría plantea tres dimensiones: el comportamiento de la tarea, el comportamiento de relación y el comportamiento del líder. El liderazgo situacional considera el comportamiento de la tarea y de la relación alta o baja, luego las combina con los 
cuatro comportamientos específicos del líder: comunicar, vender, participar, y delegar. Según el primer comportamiento, el líder define los papeles y señala a la gente cómo y cuándo debe realizar las tareas; de acuerdo con el segundo comportamiento, el líder proporciona el comportamiento directivo y de apoyo; según el tercer comportamiento, el líder y el seguidor comparten la toma de decisiones, el líder facilita y comunica; el cuarto comportamiento corresponde a un líder que proporciona poca dirección y apoyo. Este modelo propone cuatro etapas sobre la disponibilidad de los seguidores; según la primera, el seguidor es incapaz, no está dispuesto, es inseguro para asumir su responsabilidad; en la segunda, el seguidor es incapaz, está dispuesto a hacer las tareas necesarias, está motivado, carece de habilidades; en la tercera, el seguidor es muy capaz, no está dispuesto, es demasiado aprensivo para realizar lo que le indica el líder; en la cuarta, los seguidores están dispuestos y son capaces de asumir la responsabilidad.

El modelo indica que el factor más importante e influyente en la selección de estímulo de un líder es el nivel de desarrollo o madurez de un subordinado. El liderazgo depende de las exigencias que plantea una situación, donde los factores situacionales y no la herencia de la persona determinan que la persona termine imponiéndose como líder. La aparición de un gran líder depende del momento, el lugar y las circunstancias.

\section{Modelo Bass y Avolio}

El modelo de Bass (1985) tiene su origen en los conceptos fundamentales de Burns (1978), quien para muchos creó una nueva forma de entender el liderazgo. Sin embargo, no avanzó en este concepto hasta haber desarrollado una teoría clara o alguna forma de realizar mediciones.
A principios de los años ochenta, Bass inció estudios sobre temas relacionados con el liderazgo, hecho que le permitió convertir el concepto de liderazgo transformacional de Burns en una teoría rigurosa y mensurable (Sashkin, 1998).

Bass y sus colaboradores construyeron la teoría de liderazgo transformacional. Los autores consideran que este tipo de liderazgo resulta un recurso efectivo en diferentes ámbitos (Lupano y Castro, 2003). Bass distingue tres estilos de liderazgo: el laissez faire, el transaccional y transformacional.

Para medir empíricamente la conducta del líder transformacional, transaccional y laissez faire, a partir de 1985, Bass y sus colaboradores, realizaron pruebas y desarrollaron el Cuestionario Multifactorial de Liderazgo Multifactorial LeadershipQuestionnaire (MLQ). Este instrumento se ha convertido en el más utilizado para medir el liderazgo transformacional (Ramos, 2005).

Según Hunt (1999), citado en Ramos, (2005), el paradigma transformacional sacó a las corrientes tradicionales sobre liderazgo del estancamiento y de la situación en que se encontraban, considera que una de las mayores contribuciones del liderazgo transformacional, ha sido la transformación del campo de estudio del liderazgo.

\section{Liderazgo transformacional}

El liderazgo transformacional es un proceso que cambia y transforma a los individuos. Tiene una relación con valores, estándares éticos y metas a largo plazo; involucra valorar las motivaciones de los seguidores, satisfacer sus necesidades y tratarlos como seres humanos completos; además es el conjunto de capacidades que permiten al líder identificar los cam- 
bios y diseñar acciones para afrontarlo en forma efectiva (Bass, 1985). Este liderazgo considera cinco componentes o atributos claves: influencia idealizada conductual, influencia idealizada atribuida, motivación inspiracional, estimulación intelectual y consideración individualizada. Mediante estas estrategias, el líder transformacional impulsa resultados y cambios de manera directa o indirecta, influyendo en los trabajadores y formando una nueva cultura organizacional.

\section{Mayordomía de los ingresos económicos}

\section{Concepto de diezmo}

Significa la décima parte de las ganancias y entradas que el creyente destina para una finalidad sagrada. Esa décima parte es la devolución a Dios como una señal de pacto y de sociedad con él, reconociéndolo como el Creador y propietario de todas las cosas. (Génesis 14:18; Levítico 27:30 y 32; Malaquías 3:7-10)

Así también sucede con las exigencias de Dios para con nosotros. Pone sus tesoros en las manos de los hombres, pero requiere que una décima parte sea puesta fielmente a un lado para su obra. Requiere que esta porción sea entregada a su tesorería. Ha de serle devuelta como propiedad suya; es sagrada y debe emplearse para fines sagrados, para el sostén de los que han de proclamar el mensaje de salvación en todas partes del mundo.

\section{Benevolencia sistemática}

El plan de la benevolencia sistemática [el diezmo y las ofrendas] fue ordenado por Dios mismo; pero el pago fiel de lo exigido por Dios es a menudo rehusado o postergado como si las promesas solemnes no tuviesen significado. Porque los miembros de las iglesias descuidan de pagar sus diezmos y cumplir sus com- promisos, nuestras instituciones no están libres de trabas. Si todos, ricos y pobres, trajesen sus diezmos al alfolí, habría abundante provisión de recursos para aliviar la causa de trabas financieras y para llevar a cabo noblemente la obra misionera en sus diversos departamentos. Dios invita a todos los que creen la verdad a devolverle lo suyo.- Testimonios selectos, tomo 3, p. 346.

\section{Principios}

El diezmo debe usarse para el sostén del ministerio. Este pensamiento se introduce en la declaración inicial del Espíritu de Profecía recientemente citada en referencia a "aquellos que los ministraban" "en las cosas espirituales". Hay un hilo que corre, por un período de cincuenta años, a través de todos los consejos que tratan sobre dar el diezmo, como lo afirman las siguientes declaraciones de White (2008): "El diezmo es sagrado, reservado por Dios para si. Ha de ser traído a su tesorería para ser empleado en el sostén de los obreros evangélicos en su obra". Así también: "Debe dedicarse [el diezmo] únicamente para el sostén del ministerio evangélico" White (2013).

El diezmo debe traerse al "almacén" y desde allí debe distribuirse. Este principio tiene una tarea de enseñanza y un reconocimiento del dador: "Es parte de la obra del predicador [ministro] enseñar a los que aceptan la verdad por sus esfuerzos a traer el diezmo al alfolí, en reconocimiento de su dependencia de Dios" (White, 2008). Es una expresa orden: "El diezmo es sagrado, y ha sido reservado por Dios mismo. Debe ser llevado a su tesorería para que se lo emplee en la sustentación de los obreros evangélicos" (White 2013).

A diferencia de su responsabilidad en la cuestión de las ofrendas voluntarias, el 
que paga el diezmo no tiene injerencia en cuanto al lugar donde debe usarse su diezmo: "La porción que Dios se ha reservado no debe usarse para ningún otro propósito fuera del que él ha especificado. Que nadie se sienta libre para retener sus diezmos con el fin de usarlos según su propio juicio. No debe emplearse en caso de emergencia, ni como parezca conveniente, aun en cosas que conciernan a la obra de Dios" (White, 2013). No debe tener otro propósito: "Me ha sido dado un mensaje claro y bien definido para nuestro pueblo. Se me ha pedido que les comunique que están cometiendo un error al dedicar el diezmo a diferentes propósitos que, aunque son buenos en sí mismos, no son los objetivos para los cuales el Señor ha establecido el diezmo. Los que hacen este uso del diezmo se están apartando de las disposiciones del Señor. Dios los juzgará por esto". (White, 2013). No hay espacio para la infidelidad como lo demuestra White (2013): "Algunos no han estado satisfechos y han dicho: No pagaré más mi diezmo, porque no tengo confianza en la forma como se manejan las cosas en el corazón de la obra. ¿Pero robaréis a Dios porque pensáis que el manejo de la obra no es correcto? Presentad vuestras quejas en forma clara y abierta, con el espíritu debido, a las personas debidas. Pedid que las cosas sean ajustadas y puestas en orden; pero no retengáis lo que corresponde a la obra de Dios, demostrando así que sois infieles, porque otros no están obrando correctamente".

Dios tiene un plan único para todas las dispensaciones: White (2013) "El diezmo de todo lo que poseemos es del Señor. Él se lo ha reservado para que sea empleado con propósitos religiosos. Es santo. En ninguna dispensación él ha aceptado menos que esto. Un descuido o una postergación de este deber provocará el desagrado divino. Si todos los cristianos profesos llevaran sus diezmos a Dios, su tesorería estaría llena".

\section{Materiales y métodos}

El diseño de investigación es no experimental, pues los datos se recogen tal como se encuentran en el contexto de la realidad, sin ser sometidos a ninguna manipulación. Además es transversal, de tipo descriptivo correlacional, porque los datos se recogieron en un solo momento con el propósito de describir variables, y correlacionarlas, analizando la influencia de la variable independiente liderazgo, con sus respectivas dimensiones liderazgo transformacional, liderazgo transeccional y liderazgo lassiez faire en la variable dependiente mayordomía de ingresos económicos.

\section{Participantes}

Para la investigación se ha considerado la participación de los pastores distritales de la Misión Centro Oeste del Perú. La muestra fue determinada por conveniencia.

En el 2014, se contó con 35 distritos misioneros y la misma cantidad de pastores distritales; sin embargo, no todos ellos trabajaron en los años que se hizo la investigación (2012-2013). Por este motivo solo se consideró a 29 pastores que trabajaron durante el periodo 2012-2013 en la Misión. Se excluye a los pastores codificados: 6, 12, 16, 18, 24, 34 los cuales no trabajaron en la MiCOP en el periodo 2012-2013.

Los encuestados fueron 27 pastores casados, en promedio con 2 hijos, y solamente 2 pastores solteros. El promedio de edad es de 40 años, aunque se muestra una experiencia, con un promedio es de 13.8 de años de servicio. En cuanto a su funcion, atienden en promedio a 5 
iglesias y 6 grupos organizados, con un promedio de atención de miembros de la iglesia de 900 personas. Respecto de su formación profesional: 24 pastores tienen su título profesional universitario de la UPeU y 5 de instituto, a través del CESU, así como un magister y uno con grado de doctor también de la UPeU.

\section{Instrumentos}

Se aplicó el instrumento "Cuestionario Multifactorial de Liderazgo" elaborado por Bernard Bass y Bruce Avolio en la Universidad de Binghamton en Nueva York. Modificado se realizó una validación semántica por juicio de expertos. Este instrumento se aplicó en diversos países y en distintos idiomas.

Para la dimensión liderazgo transformacional se tiene 39 reactivos que recogen la información de sus componentes: influencia idealizada atribuida, influencia idealizada conductual, motivación inspira- cional, estimulación intelectual y consideración individual. Para medir el liderazgo transaccional, 18 reactivos que incluyen los tres componentes: premio contingente, administración por excepción activo y administración por excepción pasivo. Para medir el liderazgo laissez faire, se tiene 8 reactivos que recogen la información de este estilo.

\section{Resultados}

\section{Análisis descriptivos}

Los resultados de la Tabla 1 muestran que los pastores de la MiCOP presentan estilos de liderazgo transformacional, reflejando un buen porcentaje en influencia idealizada con un $89,7 \%$ en la categoria alto y muy alto, seguido de la estimulación individual con $82,7 \%$, sin embargo la influencia idealizada atribuida es la dimensión que menos porcentaje tiene en la escala alto y muy alto mostraron (41.4\%)

Tabla 1

Dimensiones de liderazgo transformacional

\begin{tabular}{|c|c|c|c|c|c|c|c|c|c|c|}
\hline \multirow{2}{*}{ Niveles } & \multicolumn{2}{|c|}{ Influencia Idealizada } & \multicolumn{2}{|c|}{$\begin{array}{c}\text { Influencia Idealizada } \\
\text { Atribuida }\end{array}$} & \multicolumn{2}{|c|}{$\begin{array}{l}\text { Motivación Inspira- } \\
\text { cional }\end{array}$} & \multicolumn{2}{|c|}{$\begin{array}{l}\text { Estimulación } \\
\text { Intelectual }\end{array}$} & \multicolumn{2}{|c|}{$\begin{array}{l}\text { Consideración } \\
\text { Individualizada }\end{array}$} \\
\hline & $\mathrm{N}$ & $\%$ & $\mathrm{~N}$ & $\%$ & $\mathrm{~N}$ & $\%$ & $\mathrm{~N}$ & $\%$ & $\mathrm{~N}$ & $\%$ \\
\hline Bajo & 1 & 3,4 & 3 & 10,3 & 1 & 3,4 & 0 & 0,0 & 1 & 3,4 \\
\hline Regular & 2 & 6,9 & 14 & 48,3 & 4 & 13,8 & 7 & 24,1 & 5 & 17,2 \\
\hline Alto & 12 & 41,4 & 8 & 27,6 & 11 & 37,9 & 12 & 41,4 & 16 & 55,2 \\
\hline Muy Alto & 14 & 48,3 & 4 & 13,8 & 13 & 44,8 & 10 & 34,5 & 7 & 24,1 \\
\hline Total & 29 & 100,0 & 29 & 100,0 & 29 & 100,0 & 29 & 100,0 & 29 & 100,0 \\
\hline
\end{tabular}

Fuente: Planillas UPeU.

Los resultados de las dimensiones del liderazgo transaccional mostrados en la Tabla 2 indican claramente que los pastores de la MiCOP trabajan con un estilo de liderazgo considerando la recompen- sa contingente: Alto $=27.6 \%$ y Muy alto $=72.4 \%$, asimismo la aplicación del estilo de liderazgo considerando la administración por excepción activa fue significativos con un $62.1 \%$, sin embargo la acep- 
ción pasiva no es manifestada por los pastores en su gran mayoría (93.1\%)

Respecto al nivel de liderazgo transformacional manifestado en los pasto- res de la MiCOP, la Tabla 3 indica que el $27,6 \%$ y el $13,8 \%$ presentaron altos niveles, lo que demuestra que los pastores de la iglesia manifestaron acciones de un líder transformador, sin embargo un 34,5\%

Tabla 2

Dimensiones de liderazgo transaccional

\begin{tabular}{ccccccc}
\hline & \multicolumn{2}{c}{ Recompensa contingente } & \multicolumn{2}{c}{ Administración por excepción activa } & \multicolumn{2}{c}{ Administración por excepción pasiva } \\
Niveles & $\mathrm{N}$ & $\%$ & $\mathrm{n}$ & $\%$ & $\mathrm{n}$ & $\%$ \\
\hline Bajo & 0 & 0,0 & 1 & 3,4 & 17 & 58,6 \\
Regular & 0 & 0,0 & 10 & 34,5 & 10 & 34,5 \\
Alto & 8 & 27,6 & 14 & 48,3 & 1 & 3,4 \\
Muy Alto & 21 & 72,4 & 4 & 13,8 & 3,4 \\
Total & 29 & 100,0 & 29 & 100,0 & 29 & 100,0 \\
\hline
\end{tabular}

Fuente: Elaboración propia.

de los mismos obtuvo porcentajes en el nivel bajo, resultado que indica que los pastores presentan diferentes estilos de liderazgo.

Tabla 3

Nivel de liderazgo transformacional de los pastores de la MiCOP

\begin{tabular}{ccc}
\hline Nivel & Frecuencia & Porcentaje \\
\hline Bajo & 10 & 34,5 \\
Regular & 7 & 24,1 \\
Alto & 8 & 27,6 \\
Muy Alto & 4 & 13,8 \\
\hline Total & 29 & 100,0 \\
\hline
\end{tabular}

Respecto al nivel de liderazgo transaccional manifestado en los pastores de la MiCOP, la Tabla 4 detalla que el $48.2 \%$ presentaron altos niveles en el estilo, lo que demuestra que los pastores de la iglesia manifestaron acciones de un líder transaccional en su mayoría; sin embargo, un $24,1 \%$ de los mismos obtuvo porcen- tajes en el nivel bajo, resultado que indica que gran parte de los pastores realizan acciones del estilo transaccional.

Tabla 4

Nivel de liderazgo transaccional de los pastores de la MiCOP

\begin{tabular}{ccc}
\hline Nivel & Frecuencia & Porcentaje \\
\hline Bajo & 7 & 24,1 \\
Regular & 8 & 27,6 \\
Alto & 7 & 24,1 \\
Muy Alto & 7 & 24,1 \\
\hline Total & 29 & 100,0 \\
\hline
\end{tabular}

Fuente: Elaboración propia.

Con respecto al estilo de liderazgo Laissez Faire, la Tabla 5 indica que 55,2\% de los pastores obtuvo un nivel regular y el $34,5 \%$ un nivel bajo, resultados que demuestra que los pastores realizan limitadas acciones respecto a este estilo de liderazgo; sin embargo, existe un 10,3\% de los mismo que aplica acciones de liderazgo Laissez Faire. 
Tabla 5

Nivel de liderazgo laissez faire de los pastores de la MiCOP

\begin{tabular}{ccc}
\hline Nivel & Frecuencia & Porcentaje \\
\hline Bajo & 10 & 34,5 \\
Regular & 16 & 55,2 \\
Alto & 2 & 6,9 \\
Muy Alto & 1 & 3,4 \\
\hline Total & 29 & 100,0 \\
\hline
\end{tabular}

Tabla 6

Estilos de liderazgo de los pastores de la MiCOP comparado con los promedios de diezmos

\begin{tabular}{cccccc}
\hline Estilo de liderazgo & $\mathrm{N}$ & $\%$ & \% diezmo 2012 & \% diezmo 2013 & Promedio \\
\hline Transformacional & 12 & $41,4 \%$ & $26,6 \%$ & $26,6 \%$ & $26,6 \%$ \\
Transaccional & 14 & $48,3 \%$ & $22,3 \%$ & $19,7 \%$ & $21,0 \%$ \\
Laissez Faire & 3 & $10,3 \%$ & $10,2 \%$ & $12,5 \%$ & $11,3 \%$ \\
\hline Total & 29 & $100,0 \%$ & & & \\
\hline
\end{tabular}

\section{Análisis inferencial}

Análisis de regresión lineal de estilo de liderazgo transformacional del pastor entre la Mayordomía de los ingresos económicos de la MiCOP (Diezmo)

Normalidad de los datos: Para determinar la normalidad de los datos se plantea la siguiente hipótesis:

Ho: Los datos de la variable en estudio se distribuyen normalmente

Ha: Los datos de la variable en estudio difieren de una distribución normal.

Los resultados en la Tabla 7 indican que las puntuaciones de las variables en estudio presentan resultados K-S estadísticamente significativos, los niveles de significación son próximos a uno $(0,869)$
Los resultado de la Tabla 6 muestra claramente que el estilo de liderazgo que predomina en los pastores de la MICOP es el transaccional con un $41.4 \%$, seguido del transformacional con un $41.4 \%$ y, finalmente, el Laissez Faire con un 10.3\%, asimismo el estilo de liderazgo que mejor resultado obtuvo fue el transformacional con un $26,6 \%$ de incremento respecto a los años 2012 y 2013, seguido del transaccional con $21,0 \%$ y el que menos resultado obtuvo es el estilo de liderazgo Laissez Faire con un 11,3\% de incremento respecto a los años mencionados, anteriormente 
yordomía de ingresos económicos de la MiCOP, 2012-2013

Ha: El liderazgo transformacional del pastor influye positivamente en la mayordomía de ingresos económicos de la MiCOP, 2012-2013

Nivel de error tipo I: El nivel de significancia es $\propto=0.05$ y por correspondiente el nivel de confianza es del $95 \%$

Regla de decision: Rechazar Ho si sig $<\alpha /$ Aceptar Ho si sig $>\alpha$

Estimación de correlación: Los resultados de la Tabla 8 explica que el análisis de correlación entre el estilo de liderazgo transformacional y la mayordomía de los ingresos económicos de la MiCOP se obtuvo una correlación positiva significativa con $r=0,468$. Asimismo el estilo de liderazgo transformacional explica la variación la mayordomía de los ingresos económicos de la MiCOP en un 21,9\%. El resultado demostró que el estilo de liderazgo transformacional contribuyó positivamente en la mayordomía de los ingresos económicos de los miembros.
Tabla 8

Prueba de Kolmogorov-Smirnov para las variables en estudio

\begin{tabular}{cccc}
\hline R & $\begin{array}{c}\mathrm{R} \\
\text { cuadrado }\end{array}$ & $\begin{array}{c}\mathrm{R} \\
\text { cuadrado } \\
\text { corregida }\end{array}$ & $\begin{array}{c}\text { Error típ. de } \\
\text { la estimación }\end{array}$ \\
\hline $0,468 \mathrm{a}$ & 0,219 & 0,190 & 0,097435 \\
\hline
\end{tabular}

a. Variables predictoras: (Constante), Puntaje de Liderazgo Transformacional

Análisis de la varianza: El resultado que muestra en la Tabla 9, determinó contrastar las hipótesis en estudio, donde, el p-valor asociado a $F_{1}($ Sig. $=0.011)$ es menor que el nivel de significación $\alpha=$ 0,05 , lo que conduce a rechazar la hipótesis nula, es decir, existe una relación lineal significativa entre liderazgo transformacional y la mayordomía de los ingresos económicos de los miembros, y dicho resultado puede estimarse en la poblacion.

Tabla 9

Análisis de Anova entre el liderazgo transformacional del pastor y la mayordomía de los ingresos económicos de los miembros

\begin{tabular}{cccccc}
\hline Modelo & $\begin{array}{c}\text { Sumade } \\
\text { cuadrados }\end{array}$ & Gl & $\begin{array}{c}\text { Media } \\
\text { cuadrática }\end{array}$ & F & Sig. \\
\hline Regresión & 0,072 & 1 & 0,072 & 7,561 & $0,011 \mathrm{a}$ \\
Residual & 0,256 & 27 & 0,009 & & \\
\hline Total & 0,328 & 28 & & & \\
\hline
\end{tabular}

a. Variables independientes: (Constante), liderazgo transformacional

Tabla 10

Análisis de estimación del liderazgo transformacional del pastor

\begin{tabular}{|c|c|c|c|c|c|}
\hline \multirow{2}{*}{ Modelo/variables } & \multicolumn{2}{|c|}{ Coeficientes no estandarizados } & \multirow{2}{*}{$\begin{array}{c}\begin{array}{c}\text { Coeficientes } \\
\text { tipificados }\end{array} \\
\text { Beta }\end{array}$} & \multirow[b]{2}{*}{$\mathrm{t}$} & \multirow[b]{2}{*}{ Sig. } \\
\hline & B & Error típ. & & & \\
\hline (Constante) & $-0,313$ & 0,196 & & $-1,599$ & 0,121 \\
\hline $\begin{array}{l}\text { X1. Liderazgo } \\
\text { transformacional }\end{array}$ & 0,004 & 0,001 & 0,468 & 2,750 & 0,011 \\
\hline
\end{tabular}

Regla de decisión: Como el valor sig. $=0.011<\propto=0.05$, se rechaza $\mathrm{H} \_0$, a favor de la $\mathrm{H} \_$a; declarando que el liderazgo transformacional contribuyó positivamente la mayordomía de los ingresos económicos de la MiCOP.

Análisis de estimación: La Tabla 9 evidencia que el liderazgo transformacional contribuye positivamente a la mayordomía de los ingresos económicos de la Mi$\operatorname{COP}(p<0,05)$.
Conclusión: Se concluye que el estilo de liderazgo transformacional se relaciona directamente con la mayordomía de los ingresos económicos de la MiCOP $(p<0,05)$; la variación de la mayordomía 
de los ingresos económicos de la MiCOP en un $21,9 \%$ a causa del estilo de liderazgo transformacional de los pastores de la MiCOP.

Análisis de regresión lineal de estilo de liderazgo transaccional del pastor entre la mayordomía de los ingresos económicos de la MiCOP (Diezmo)

Normalidad de los datos: Para determinar la normalidad de los datos se plantea las siguientes hipótesis:

Ho: Los datos de la variable en estudio se distribuyen normalmente

Ha: Los datos de la variable en estudio difieren de una distribución normal

Los resultados en la tabla 11 indican que las puntuaciones de las variables en estudio presentan resultados K-S estadísticamente significativos, los niveles de significación son próximos a uno $(0,368)$ por encima del $\alpha=0.05$ lo que permite rechazar la hipótesis alterna y aceptar la nula, concluyendo que los datos se distribuyen normalmente de manera que es necesario la utilización de análisis estadístico paramétrico

Tabla 11

Prueba de Kolmogorov-Smirnov para las variables en estudio

\begin{tabular}{lc}
\hline & Standardized Residual \\
\hline $\mathrm{N}$ & 29 \\
Z de Kolmogorov-Smirnov & 0,918 \\
Sig. asintót. (bilateral) & 0,368 \\
\hline
\end{tabular}

Variables de análisis: La variable dependiente (y) es la mayordomía de los ingresos económicos (Diezmo) y la variable independiente $(\mathrm{x})$ es el estilo de liderazgo transaccional del pastor

\section{Formulación de las hipótesis}

Ho: El liderazgo transaccional del pastor no influye positivamente en la mayordomía de ingresos económicos de la Mi-
COP, 2012-2013

Ha: El liderazgo transaccional del pastor influye positivamente en la mayordomía de ingresos económicos de la MiCOP, 2012-2013

Nivel de error tipo I: El nivel de significancia será $\propto=0.05$ y por correspondiente el nivel de confianza es del $95 \%$

Regla de decisión: Rechazar Ho si sig $<\alpha /$ Aceptar Ho si sig $>\alpha$

Estimación de correlación: Los resultados de la Tabla 12 explican que del análisis de correlación entre el estilo de liderazgo transaccional y la mayordomía de los ingresos económicos, obtuvo una correlación positiva considerable con $r$ $=0,499$. Asimismo el estilo de liderazgo transaccional explica la variación la mayordomía de los ingresos económicos en un $24,9 \%$. El resultado demostró que el estilo de liderazgo transaccional contribuyó positivamente en la mayordomía de los ingresos económicos de la MiCOP.

Tabla 12

Análisis de correlación entre el liderazgo transaccional del pastor y la mayordomía de los ingresos económicos de los miembros

\begin{tabular}{lcccc}
\hline Modelo & $\mathrm{R}$ & $\begin{array}{c}\mathrm{R} \\
\text { cuadrado }\end{array}$ & $\begin{array}{c}\mathrm{R} \\
\text { cuadrado } \\
\text { corregida }\end{array}$ & $\begin{array}{c}\text { Error típ. de la } \\
\text { estimación }\end{array}$ \\
\hline 1 & $0,499 a$ & 0,249 & 0,221 & 0,095548 \\
\hline $\begin{array}{l}\text { a. Variables predictoras: (Constante), Puntaje de Liderazgo } \\
\text { Transaccional }\end{array}$
\end{tabular}

Análisis de la varianza: El resultado mostrado en la Tabla 13, determinó contrastar las hipótesis en estudio, donde, el p-valor asociado a F, (Sig. $=0.006)$ es menor que el nivel de significación $\alpha=$ 0,05 , lo que conduce a rechazar la hipótesis nula, es decir, existe una relación lineal significativa entre liderazgo transaccional y la mayordomía de los ingresos económicos de los miembros. 
Tabla 13

Análisis de ANOVA entre el liderazgo transaccional del pastor y la mayordomía de los ingresos económicos de los miembros

\begin{tabular}{cccccc}
\hline Modelo & $\begin{array}{c}\text { Sumade } \\
\text { cuadrados }\end{array}$ & gl & $\begin{array}{c}\text { Media } \\
\text { cuadrática }\end{array}$ & $F$ & Sig. \\
\hline Regresión & 0,082 & 1 & 0,082 & 8,940 & $0,006 a$ \\
Residual & 0,246 & 27 & 0,009 & & \\
Total & 0,328 & 28 & & & \\
\hline
\end{tabular}

a. Variables independientes: (Constante), Liderazgo Transaccional
Análisis de estimación: La tabla 14 evidencia que el liderazgo transaccional contribuye positivamente a la mayordomía de los ingresos económicos $(p<0,05)$.

Regla de decisión: Como el valor sig. $=0.006<\propto=0.05$, se rechaza $\mathrm{H}_{-} 0$, a favor de la H_a; declarando que el Liderazgo transaccional contribuyó positivamente la mayordomía de los ingresos económicos de la MiCOP.

Tabla 14

Análisis de estimación del liderazgo transaccional del pastor

\begin{tabular}{ccccccc}
\hline \multirow{2}{*}{ Modelo/variables } & \multicolumn{2}{c}{ Coeficientes no estandarizados } & Coeficientes tipificados & & Sig. \\
\cline { 2 - 5 } & B & Error típ. & Beta & & $-0,282$ & 0,780 \\
(Constante) & $-0,024$ & 0,085 & & 2,990 & 0,006 \\
\hline
\end{tabular}

Conclusión: Se concluye que el estilo de liderazgo transaccional se relaciona directamente con la mayordomía de los ingresos económicos ( $p<0,05)$; siendo explicada la variación la mayordomía de los ingresos económicos en un $24,9 \%$ a causa del estilo de liderazgo transaccional de los pastores de la MiCOP.

Análisis de regresión lineal de estilo de liderazgo Laissez Faire del pastor entre la Mayordomía de los ingresos económicos (Diezmo)

Normalidad de los datos: Para determinar la normalidad de los datos se plantea la siguiente hipótesis:

Ho: Los datos de la variable en estudio se distribuyen normalmente

Ha: Los datos de la variable en estudio difieren de una distribución normal

Los resultados presentados en la $\mathrm{Ta}$ bla 15 indican que las puntuaciones de las variables en estudio presentan resul- tados K-S estadísticamente significativos, los niveles de significación son próximos a uno $(0,330)$ por encima del $\alpha=0.05$, lo que permite rechazar la hipótesis alterna y aceptar la nula, concluyendo que los datos se distribuyen normalmente de manera que es necesario la utilización de análisis estadístico paramétrico.

Tabla 15

Prueba de Kolmogorov-Smirnov para las variables en estudio

\begin{tabular}{lc}
\hline & Standardized Residual \\
\hline $\mathrm{N}$ & 29 \\
Z de Kolmogorov-Smirnov & 0,948 \\
Sig. asintót. (bilateral) & 0,330 \\
\hline
\end{tabular}

Variables de análisis: La variable dependiente (y) es la mayordomía de los ingresos económicos (Diezmo) y la variable independiente $(\mathrm{x}$ ) es el estilo de liderazgo transaccional del pastor

Variables de análisis: La variable dependiente (y) es la mayordomía de los ingresos económicos (Diezmo) y la ariable independiente $(x)$ es el estilo de liderazgo laissez faire del pastor 


\section{Formulación de las hipótesis}

Ho: El liderazgo laissez faire del pastor no influye positivamente en la mayordomía de ingresos económicos de la MiCOP, 2012-2013

Ha: El liderazgo laissez faire del pastor influye positivamente en la mayordomía de ingresos económicos de la MiCOP, 2012-2013

Nivel de error tipo I: El nivel de significancia será $\propto=0.05$ y por correspondiente el nivel de confianza es del 95\%

Regla de decisión: Rechazar Ho si sig $<\alpha /$ Aceptar Ho si sig $>\alpha$

Estimación de correlación: Los resultados de la Tabla 16 explica que el análisis de correlación entre el estilo de liderazgo laissez faire y la mayordomía de los ingresos económicos se obtuvo una correlación negativa considerable con $r=$ $-0,405$. Asimismo el estilo de liderazgo transformacional explica la variación la mayordomía de los ingresos económicos en un $16,4 \%$. El resultado demostró que el estilo de liderazgo laissez faire contribuyó negativamente en la mayordomía de los ingresos económicos de la MiCOP.
Tabla 16

Análisis de correlación entre el liderazgo laissez faire del pastor y la mayordomía de los ingresos económicos de los miembros

\begin{tabular}{cccc}
\hline$R$ & R cuadrado & $\begin{array}{c}\text { R cuadrado } \\
\text { corregida }\end{array}$ & $\begin{array}{c}\text { Error típ. de la } \\
\text { estimación }\end{array}$ \\
\hline$-0,405 a$ & 0,164 & 0,133 & 0,100804 \\
\hline
\end{tabular}

a. Variables predictoras: (Constante), puntaje de liderazgo laissez faire

Análisis de la varianza: El resultado de la Tabla 17 determinó contrastar las hipótesis en estudio, donde, el p-valor asociado a $F$, (Sig. $=0.0029$ ) es menor que el nivel de significación $\alpha=0,05$, lo que conduce a rechazar la hipótesis nula, es decir existe una relación lineal inversa significativa entre liderazgo laissez faire y la mayordomía de los ingresos económicos de los miembros.

Tabla 17

Análisis de ANOVA entre el liderazgo transaccional del pastor y la mayordomía de los ingresos económicos de los miembros

\begin{tabular}{cccccc}
\hline Modelo & $\begin{array}{c}\text { Sumade } \\
\text { cuadrados }\end{array}$ & gl & $\begin{array}{c}\text { Media } \\
\text { cuadrática }\end{array}$ & F & Sig. \\
\hline Regresión & 0,054 & 1 & 0,054 & 5,289 & $0,029 a$ \\
Residual & 0,274 & 27 & 0,010 & & \\
Total & 0,328 & 28 & & & \\
\hline
\end{tabular}

a. Variables independientes: (Constante), liderazgo laissez faire

Análisis de estimación: La Tabla 19 evidencia que el Liderazgo Laissez Faire contribuye negativamente a la mayordomía de los ingresos económicos $(p<0,05)$.

Tabla 19

Análisis de estimación del liderazgo laissez faire del pastor

\begin{tabular}{ccccccc}
\hline \multirow{2}{*}{ Modelo/variables } & \multicolumn{2}{c}{ Coeficientes no estandarizados } & Coeficientes tipificados & & S & Sig. \\
\cline { 2 - 5 } & $\mathrm{B}$ & Errortíp. & Beta & & 5,370 & 0,000 \\
(Constante) & 0,380 & 0,071 & $-0,405$ & $-2,300$ & 0,029 \\
\hline
\end{tabular}

Regla de decision: Como el valor sig. $=0.002<\propto=0.05$, se rechaza $\mathrm{H}_{0}$, a favor de la $\mathrm{H}_{\mathrm{a}}$; declarando que el liderazgo lais- sez faire contribuyó negativamente a la mayordomía de los ingresos económicos de la MiCOP. 
Conclusión: Se concluye que el estilo de liderazgo Laissez Faire se relaciona inversamente con la mayordomía de los ingresos económicos $(p<0,05)$; siendo explicada la variación la mayordomía de los ingresos económicos en un 16,4\% a causa del estilo de liderazgo Laissez Faire de los pastores de la MiCOP.

\section{Conclusión}

Como conclusión general se asume que la satisfacción de los trabajadores es la causa de la satisfacción de los clientes en la Universidad Peruana Unión en el año 2007. De 270 trabajadores investigados, el 55\% expresó estar insatisfecho en su trabajo actual y de 615 estudiantes investigados (clientes directos), el 50\% expresó estar insatisfecho con el tipo de servicio recibido en la universidad.

Los trabajadores administrativos y docentes de la Universidad Peruana Unión están insatisfechos intrínsecamente en su actual trabajo. El $51 \%$ de una muestra de 270 investigados expresó su insatisfacción en el aspecto intrínseco. Los factores considerados para determinar la satisfacción intrínseca son: oportunidades de promoción laboral, nivel de coherencia de la especialidad que tiene con el puesto de trabajo asignado, nivel de coherencia de la experiencia que tiene con el trabajo que realiza, dinámica del trabajo que realiza, es decir, es monótono o motivador, medida en que considera que sus expectativas personales se satisfacen con el trabajo que realiza.
Los trabajadores administrativos y docentes de la Universidad Peruana Unión expresaron su insatisfacción en su actual trabajo en la dimensión extrínseca. El $60 \%$ de una muestra de 270 investigados expresó su insatisfacción en el aspecto extrínseco: estatus o puesto laboral en el que se encuentra. Los factores considerados para determinar la satisfacción intrínseca son: seguridad y condiciones de trabajo, nivel de remuneración que recibe mensualmente, tipo de relación con los compañeros de trabajo, tipo de gestión de personal de las autoridades universitarias.

Los clientes (alumnos) de las tres sedes de la Universidad Peruana unión expresan su insatisfacción con el servicio que les brinda su universidad durante el año académico de 2007. De una muestra de 615 estudiantes investigados, el 50\% expresó no estar satisfecho con el actual servicio que les brinda su institución donde se forman profesionalmente. Las áreas consideradas para determinar la satisfacción del cliente son: efectividad en el trámite de documentos, efectividad en la atención directa del cliente y gestión de personal (área administrativa); estructura curricular, desempeño didáctico de los docentes y aprendizajes logrados (área académica); transporte, internet, comedor estudiantil y asistencia social (área de bienestar y servicio social). 


\section{Referencias}

Alcázar-Zamacona J., \& Haro-Zea K. (2009). Estilo de liderazgo predominante en docentes universitarios en México, Universidad Popular Autónoma del Estado de Puebla. Global Conference on Business and Finance Proceedings, Volume 9, Number 2.

Bass, B. (1985). Leadership and performance beyond expectations. New York: The Free Press.

Bass, B. (1998). Transformational leadership. Industrial, military and educational impact. Nueva Jersey: Lawrence Erlbaum Associates.

Bass, B. (1990). Bass and Stogdill's handbook of leadership: Theory, research and managerial applications, (3ra. ed.). New York, NY: The Free Press.

Bass, B. \& Avolio, B. (1990). Manual for the multifactor leadership questionnaire. Palo Alto, CA: Consulting Psychologists Press.

Bass, B. \& Avolio, B. (1994). Improving organizational effectiveness. Through transformational leadership. Thousand Oaks, CA: Sage Publications.

Bass, B. M., \& Riggio, R. E. (2006). Transformational leadership. Mahwah, NJ: Lawrence Erlbaum Associates.

Bernal, A. (2000). Metodología de la investigación para administración y economía. Colombia: Pearson Educación de Colombia, Ltda.

Brown, D. (2005). Elaborating the construct of transformational leadership: The role of affect. Leadership Quarterly.Vol. 16 Issue 2, pp. 245-272.
Carrera, B. (2002). Tendencias del liderazgo en el docente venezolano de la I etapa de educación básica. Investigación y Postgrado. Vol. 17. Num. 2. Recuperado en la dirección electrónica www.scielo.org. ve/scielo.php

Chiavenato, I. (1999). Introducción a la teoría general de administración. Colombia: Mc Graw Hill.

Chiavenato, I. (2002). Administración en los nuevos tiempos. Colombia: Mc Graw Hill.

Concepción, Alanis, Lerma. (2014). Estilos de liderazgo de los directivos del CIIDIR DURANGO y la percepción de su eficacia desde el MLRC. Global Conference on Business and Finance Proceedings, Volume 9, Number 2, Universidad Juárez del Estado de Durango

Covey, S. (2005). Los siete hábitos de la gente altamente efectiva. España. Ediciones Paidós Ibérica, S.A

Covey, S. (2005). El 8th hábito: de la efectividad a la grandeza. España. Ediciones Paidós Ibérica, S.A

Cuadra, A. \& Veloso, C. (2007). Liderazgo, clima y satisfacción laboral en las organizaciones. Revista Universum, 22(2), 40-56.

Drucker, P. (2002). La gerencia en la sociedad futura. New York: Edit. Norma.

Epitropaki, O. \& Martin, R. (2005). A Longitudinal study of the role of implicit Leadership theories Exchange and Employee Outcomes. Journal of Applied Psichology. Vol. 90 Issue 4, p 659-676.

Faten, F. (2004). Transactional versus transformational style of leadership.Quality Management in Health Care. Vol. 13, p274-284, 7p, 3 charts. 
Fernández San Martín, M. (2000). Job satisfaction among primary care professionals in Area 10 Insalud, Madrid. Revista Especializada Salud Pública. Recuperado de http://www.scielo.isciii.es/scielo.php

Ferreiro, P. \& Alcázar, M. (2001). Gobierno de personas en la empresa. Universidad de Piura: PAD- Escuela de Dirección.

Ferrer, J. Clemenza C. \& Romero Rojas L. (2000). Liderazgo corporativo y crisis paradigmático universitario. Universidad de Zulia Maracaibo Venezuela. Recuperado de http://redalyc.uaemex.mx

Fischman, D. (2005). El líder transformador I. Universidad Peruana de Ciencias Aplicadas. Empresa Editora El Comercio S.A.

Gonzales, P. (2004). Equipos de trabajo y su influencia en la gestión eficaz de las empresas del grupo empresarial Unión. Universidad Nacional Federico Villarreal, Lima - Perú.

Goris, J. R. (1999). Un modelo de liderazgo en el umbral del Siglo XXI: La nueva ciencia. División de Postgrado, Universidad de Montemorelos.

Great Place to Work Institute Perú. Las 25 mejores empresas para trabajar en el Perú. Diario El Comercio del 29 de noviembre del 2006.

Heifetz, R. \& Laure, D. (1997). El trabajo del liderazgo. Leadership without easy anwsers. Harvard University.

Hernández, R. (2006). Metodología de la Investigación. México. McGraw-Hill.

Huamaní, Gloria. (2006). Liderazgo y productividad desde la perspectiva de gestión de conocimiento y dirección estratégica. Universidad Nacional Federico Villa- rreal - Escuela Universitaria de Postgrado. Lima-Perú.

Krames, J. (2006). Jack Welch y las 4E del liderazgo. México: Editorial Aguilar.

Koontz, H. (1998). Administración una perspectiva global. México: McGraw Hill.

Lussier, R. \& Achua. (2002). Liderazgo. México. Internacional Thomson Editores S.A.

Mcwhirter, J. (2000). Liderazgo sistémico. Una aplicación del development behavioural Modelling. Recuperado en la dirección electrónica www.scsystemic.com/ lidersist.htm). Julio-2005.

Méndez, C. (2004). Metodología diseño y desarrollo del proceso de investigación. Colombia. McGraw Hill.

Mendoza, I. \& Torres, J.R. (2006, noviembre). Percepción del perfil de liderazgo transformacional y transaccional de gerentes de ventas de empresa químico farmacéutica de clase mundial en México, por parte de sus trabajadores inmediatos. Actas del IV Congreso Internacional de Análisis Organizacional. Jalapa, Veracruz, México.

Nancy L. M. (2005). Transformational leadership in human service organizations. Administration in Social Work. Vol. 29 Issue 2, p105-118, 14p.

Nir, A. (2005). School-Parents Relationship in the Era of School-Based Management: Harmony or Conflict? Leadership \& Policy in Schools. Vol. 4 Issue 1, p55-72, $18 \mathrm{p}$.

Palomo, M. (2005). Liderazgo. y motivación de equipos de trabajo Madrid: ESIC Edito91 rial. 
Pedraja, L., Rodríguez, E. \&Rodríguez, J. (2006). Liderazgo y decisiones estrategicas: Una perspectiva integradora. Asociación Interciencia. Vol. 31 Num 008. Recuperado el 20 de octubre del 2006, de la base de datos Redalyc.

Pedraja-Rejas, L., Rodríguez-Ponce, E. \& Rodríguez-Ponce, J. (2008). Importancia de los estilos de liderazgo sobre la eficacia: un estudio comparativo entre grandes y pequeñas y medianas empresas privadas www.researchgate. net/publication/40904960/file/32bfe51470d33c774d.pdf

Pedraja-Rejas, L.; Rodríguez-Ponce; E.; Barreda Olavarría, M., Sagredo Núñez, O. \& Segovia León C. (2009). Estilos de liderazgo y resultados del sistema de medición de la calidad de la educación: Un estudio empírico en los colegios básicos de la ciudad de Arica-Chile. Ingeniare. Revista Chilena de Ingeniería, 17 (1), pp. 21-26

Robbins, Stephen y Coulter, Mary. (2000). Administración. México: Prentice Hall.

Robbins, S. P. (1999). Comportamiento organizacional. México: Prentice Hall.

Senge, P. (1998). La quinta disciplina. México: Granica.

Sepulveda, A. \& Cisterna, J. (2001). Razones relacionadas con la satisfacción docente. Revista Electrónica Diálogos Educativos. Num. 4, Art. 6.
Sierra. (1995). Tesis doctorales y trabajos de investigación científica. España. Editorial Paraninfo S.A.

Siliceo, A. (2001). Liderazgo: el don del servicio. Mexico: McGraw-Hill.

Universidad Nacional de San Luis, Argentina. (2004). El síndrome de Burnout y sus posibles consecuencias en el trabajador docente. Recuperado en: http://redalyc.uaemex.mx/redalyc/scr/inicio

Thieme, C.P. (2005). Liderazgo y eficiencia en la educación primaria: El caso de Chile. Tesis doctoral, Universidad Autónoma de Barcelona, España.

Urch, V. \& Wheeler, J. (2003). Managing from the boundary: The Effective Leadership of Self-Managing work teams. Academy of Management Journal. Vol.46. No. 4. 435-457.

White, Elena G. de. (2013). Consejos sobre mayordomía cristiana. 3ra. Edic. Florida, Argentina: ACES.

White, Elena G. de. (2013). Obreros evangéli$\cos 2$ da. Edic. Florida, Argentina: ACES

Zaleznik, A. (1992). Directivos y lideres: ¿son diferentes? Harvard Bussiness Review. 